Chapman University

Chapman University Digital Commons

Physical Therapy Faculty Articles and Research

Physical Therapy

11-26-2009

The Impact of Left Hemisphere Stroke on Force Control with Familiar and Novel Objects: Neuroanatomic Substrates and Relationship to Apraxia

Amanda M. Dawson

Laura J. Buxbaum

Susan V.Duff

Follow this and additional works at: https://digitalcommons.chapman.edu/pt_articles

Part of the Movement and Mind-Body Therapies Commons, Musculoskeletal System Commons, Other Rehabilitation and Therapy Commons, Physical Therapy Commons, and the Physiotherapy Commons 


\title{
The Impact of Left Hemisphere Stroke on Force Control with Familiar and Novel Objects: Neuroanatomic Substrates and Relationship to Apraxia
}

\author{
Comments \\ NOTICE: this is the author's version of a work that was accepted for publication in Brain Research. Changes \\ resulting from the publishing process, such as peer review, editing, corrections, structural formatting, and \\ other quality control mechanisms may not be reflected in this document. Changes may have been made to this \\ work since it was submitted for publication. A definitive version was subsequently published in Brain Research, \\ volume 4, in 2010. DOI: 10.1016/j.brainres.2009.11.034 \\ The Creative Commons license below applies only to this version of the article.
}

\section{Creative Commons License}

\section{(c) 1 (1) 90}

This work is licensed under a Creative Commons Attribution-Noncommercial-No Derivative Works 4.0 License.

\section{Copyright}

Elsevier 


\title{
THE IMPACT OF LEFT HEMISPHERE STROKE ON FORCE CONTROL WITH FAMILIAR AND NOVEL OBJECTS: NEUROANATOMIC SUBSTRATES AND RELATIONSHIP TO
}

\section{APRAXIA}

\author{
Amanda M. Dawson ${ }^{*}$, Laurel J. Buxbaum*, and Susan V. Duff ${ }^{\dagger}$ \\ "Moss Rehabilitation Research Institute, Philadelphia, USA \\ †Department of Physical Therapy, Thomas Jefferson University, Philadelphia, USA
}

\begin{abstract}
Fingertip force scaling for lifting objects frequently occurs in anticipation of finger contact. An ongoing question concerns the types of memories that are used to inform predictive control. Objectspecific information such as weight may be stored and retrieved when previously encountered objects are lifted again. Alternatively, visual size and shape cues may provide estimates of object density each time objects are encountered. We reasoned that differences in performance with familiar versus novel objects would provide support for the former possibility. Anticipatory force production with both familiar and novel objects was assessed in 6 left hemisphere stroke patients, 2 of whom exhibited deficient actions with familiar objects (ideomotor apraxia; IMA), along with 5 control subjects. In contrast to healthy controls and stroke participants without IMA, participants with IMA displayed poor anticipatory scaling with familiar objects. However, like the other groups, IMA participants learned to differentiate fingertip forces with repeated lifts of both familiar and novel objects. Finally, there was a significant correlation between damage to the inferior parietal and superior and middle temporal lobes, and impaired anticipatory control for familiar objects. These data support the hypotheses that anticipatory control during lifts of familiar objects in IMA patients are based on object-specific memories, and that the ventro-dorsal stream is involved in the long-term storage of internal models used for anticipatory scaling during object manipulation.
\end{abstract}

\section{Keywords}

stroke; apraxia; ipsilesional hand; internal model; grasp; motor planning

\section{INTRODUCTION}

Smooth and stable lifts of objects depend on memory representations that capture the relationship between physical properties of objects such as weight, the force requirements to lift the object, and the dynamics and mechanics of the sensorimotor system. Such memory

\section{(C) 2009 Elsevier B.V. All rights reserved.}

Laurel J. Buxbaum or Amanda Dawson, Moss Rehabilitation Research Institute, 1200 W. Tabor Rd., Philadelphia, PA 19141, lbuxbaum@einstein.edu, dawsona@einstein.edu.

Publisher's Disclaimer: This is a PDF file of an unedited manuscript that has been accepted for publication. As a service to our customers we are providing this early version of the manuscript. The manuscript will undergo copyediting, typesetting, and review of the resulting proof before it is published in its final citable form. Please note that during the production process errors may be discovered which could affect the content, and all legal disclaimers that apply to the journal pertain. 
representations, frequently characterized as "internal models", are used for anticipatory control during object lifts to prevent slippage and object deformation. By using predictions about object properties based on prior experience, anticipatory grip force $(\mathrm{GF})^{1}$ and vertical load force (LF) enhance lift coordination (Flanagan \& Tresilian, 1994;Wolpert \& Flanagan, 2001;Kawato et al., 2003).

Anticipatory fingertip force deficits have been described in conjunction with several central nervous system disorders (Nowak, Timmann \& Hermsdörfer, 2007; Duff \& Gordon, 2003) but to this point have not explicitly been identified in stroke populations (Nowak, Grefkes et al., 2007). It is notable, however, that participants with a single-hemisphere stroke exhibit slow, inaccurate grips, and use excessive or variable grip force scaling bilaterally (Quaney, Perera, Maletsky, Luchies \& Nudo, 2005; Blennerhassett, Carey \& Matyas, 2006). Such abnormalities might well be attributable, at least in part, to a deficit in anticipatory planning.

Most previous studies with adults post-stroke have solely assessed performance with novel geometric objects, such as cylinders or cubes, (except see Gordon, Westling, Cole, \& Johansson, 1993; Duff \& Gordon, 2003), limiting their ecological relevance. Additionally, they have either failed to assess for the presence of ideomotor apraxia (IMA) (Nowak, Hermsdörfer \& Topka, 2003; Blennerhassett et al., 2006), or alternatively, have excluded participants who exhibited it (Raghavan, Krakauer \& Gordon, 2006; Quaney et al., 2005; Nowak, Grefkes et al., 2007; Dafotakis et al., 2008; but see Li, Randerath, Goldenberg \& Hermsdörfer, 2007, for an exception). This appears to be a critical omission given that IMA, a disorder of complex object-related action observed in both the contralesional as well as ipsilesional hand, is a frequent consequence of stroke, particularly to the brain's left hemisphere. Individuals with IMA are deficient in anticipatory planning of hand posture and hand orientation (Buxbaum, Johnson-Frey \& Bartlett-Williams, 2005) and rely upon visual feedback during action imitation (Jax, Buxbaum \& Moll, 2006) and in reaching to targets (Haaland, Harrington \& Knight, 1999). Therefore, IMA may reflect, at least in part, a deficit in storage or retrieval of internal models used for anticipatory control of object manipulation.

An additional relevant feature of IMA is a surprising disparity between actions to familiar versus novel objects in favor of the latter. For example, when tested with the ipsilesional hand, participants with IMA have difficulties recognizing and performing the correct hand postures for familiar objects, but perform normally on the same tasks with novel objects (Buxbaum, Sirigu, Schwartz \& Klatzky, 2003), and are more impaired in imitation of the hand posture component of meaningful, as compared to meaningless, object-related actions (Buxbaum, Kyle, Grossman \& Coslett, 2007). This suggests that retrieval of stored representations may in some cases disrupt spatiomotor processing in IMA.

We postulated that the study of anticipatory force scaling during lifts of novel and familiar objects in participants with IMA would provide evidence relevant to the question of the nature of memory representations used for anticipatory force control. An ongoing question in the literature concerns the types of memories that are used for anticipatory control during object manipulation. There are several candidate theories. One possibility is that object-specific information such as weight is stored, either as part of an internal model or as an independent

\footnotetext{
${ }_{1}^{1}$ ABBREVIATIONS: GF, grip force; LF, load force; IMA, ideomotor apraxia; LCVA, left cerebrovascular accident; GTS, percentage of total possible points on the Gesture to Sight of Objects apraxia measure; MTG, middle temporal gyrus; STG, superior temporal gyrus; TO, posterior temporo-occipital; IPL, inferior parietal lobe; IFG, inferior frontal gyrus; PM, Premotor cortex; GFR, first peak grip force rate; LFR, first peak load force rate; GFR Order, percentage of correctly ordered GFRs; LFR Order, percentage of correctly ordered LFRs; GFR Distance, sum of GFR inter-item distance scores; LFR Distance, sum of LFR inter-item distance scores; Monofil, monofilament test; 2 pt, static 2-point discrimination in mm; GrStr, hand grip strength in lbs; Peg, grooved pegboard; MobergTtl, Moberg test total pickup time in s; MobergID, Moberg item identification time, in s; WAB AC Western Aphasia Battery auditory comprehension subtest.
} 
neural representation. Thus, when previously encountered objects are to be lifted again, the object-specific information is retrieved to scale the GF and LF needed for successful manipulation. Consistent with this possibility, healthy participants are able to scale load and grip forces to object weight on their first lift with familiar objects, but only after several lifts of a novel object when size-weight cues are absent (Gordon et al., 1993). An additional possibility is that visual size and shape cues allow for an estimation of object density every time an object is encountered (Mon-Williams \& Murray, 2000; Quaney, Rotella, Peterson, \& Cole, 2003; Cole, 2008). There is evidence, for example, that predictive fingertip forces are adjusted when objects of constant density vary in size (Gordon, Forssberg Johansson \& Westling, 1991). Thus, smaller objects are perceived as heavier than larger objects of the same weight, (the "size-weight illusion"), indicating that visual size cues are influential in initially estimating object weight until sensory feedback adjusts that estimate (Flanagan \& Beltzner, 2000; Grandy \& Westwood, 2006).

The influence of object-specific versus current visual information in anticipatory fingertip force scaling may be assessed by examining performance during lifts of familiar versus novel objects in healthy as well as in neurologic participants with suspected anticipatory control deficits. If different representations and processes contribute to lifting familiar as compared to novel objects (e.g., long-term versus short-term memories), then performance should differ for the two object types. Alternatively, if the same mechanisms and representations (such as the use of visual size cues to estimate density) are used for both object types, then performance should be parallel in the two cases.

The final goal of the study was to assess the left hemisphere lesion loci related to anticipatory control deficits during lifts of familiar and novel objects. In our previous work in individuals with IMA, we proposed that the behavioral distinction between hand postures associated with familiar versus unfamiliar objects is seated in a corresponding functional neuroanatomic distinction between two processing streams specialized for actions upon objects: a bilateral fronto-parietal system forming the dorso-dorsal stream, specialized for online control of visually-guided action based on object geometry (size, shape); and a left-lateralized inferior parietal lobe (IPL) and superior temporal gyrus (STG) system forming the ventro-dorsal stream, specialized for stored object-related actions (Buxbaum, 2001; Rizzolatti \& Matelli, 2003; Buxbaum, Frey et al., 2005; Frey, 2007). IMA patients with lesions to the "object use" system (but not "object grasp" system) have deficits not only in the production of object-related actions, but in their recognition as well, suggesting that a common representational deficit may underlie both impairments (Buxbaum, Kyle, \& Menon, 2005; Buxbaum et al., 2007). In parallel, numerous functional neuroimaging studies have demonstrated left IPL and posterior STG activation when subjects observe or plan familiar tool-use movements (e.g., JohnsonFrey, 2004; Lewis, 2006; Creem-Regehr \& Lee, 2005), as well as when they plan object-related actions based on immediate or final goals (Majdandzic et al., 2007).

In light of the two route theory, and the associated evidence on the role of the IPL and STG in action planning, it is reasonable to predict that structures that form the ventro-dorsal route of the left hemisphere may be implicated in anticipatory planning of force control with objects. Functional imaging studies suggest the left IPL may contribute to the selection of grip force appropriate to a given object (Ehrsson, Fagergren, \& Forssberg, 2001; Kuhtz-Bushbeck, Ehrsson \& Forssberg, 2001), while the STG has not been implicated in grip or load force control and thus its role remains an open question.

Based on previous research, we were able to make a number of behavioral and neuroanatomic predictions in the present study. We expected that participants with left hemisphere lesions and IMA would generate impaired predictive forces on the first attempt to lift familiar objects as compared to novel objects. We also predicted anticipatory control during lifts of familiar 
objects to be dependent on lesion location, where lesions to the left IPL and STG would be most disruptive to established representations, resulting in poor performance on the first lift with familiar objects.

\section{METHODS}

\section{Participants}

Participants were 6 right-handed individuals who had suffered a single, left hemisphere cerebrovascular accident (LCVA), and 5 right-handed healthy age and education matched controls (age: $\mathrm{t}(9)=-.94, \mathrm{p}>.05$; education: $\mathrm{t}(9)=-.56, \mathrm{p}>.05$ ). All LCVA participants sustained their stroke at least three months prior to the initiation of testing (mean $=7.5$ years, see Table 1). LCVA participants were selected from a research database based on availability, representation of a range of IMA scores, and the ability to travel to Thomas Jefferson University for testing.

Participants were excluded if ipsilesional (left) grip strength did not fall within two standard deviations of published norms for their age and gender (Mathiowetz et al., 1985). Participants were identified as exhibiting IMA when their average score on 10 trials of pantomiming gestures to the sight of objects (GTS) fell two standard deviations below the mean of healthy control performance (control mean score $=95, \mathrm{sd}=4.68$; previously reported in Buxbaum, Frey et al., 2005). In the GTS task, objects are presented singly and participants are asked to pantomime how to use each object with their ipsilesional hand (see Buxbaum, Kyle et al., 2005 , for details). Based on this criterion, 2 patients were characterized as exhibiting IMA (See Table 1).

All participants consented to the study in accordance with the guidelines of the Albert Einstein Healthcare Network and Thomas Jefferson University Institutional Review Boards and were paid for their participation.

\section{Clinical Data}

Each subject was tested using standard clinical assessments of sensibility, manual dexterity, and grip strength for each hand (See Table 1). Passive sensibility was assessed via static, twopoint discrimination and pressure sensitivity using the Semmes-Weinstein Monofilament Test ( $\log _{10}, .1 \mathrm{mg}$; Semmes, Weinstein, Ghent \& Teuber, 1960;Stone, 1992;Weber, 1935). Active sensibility was tested with the modified Moberg pickup test of tactile gnosis (Dellon, 1980;Moberg, 1958). Manual dexterity was tested using the timed 9-hole peg test (Mathiowetz et al., 1992). Gross grip strength was assessed using the Jamar Dynamometer (Bechtol, 1954). Language comprehension was assessed with the Comprehension subtest of the Western Aphasia Battery (WAB; REF).

\section{Neuroanatomic Scan Acquisition and Segmentation}

We obtained high-resolution structural MRI scans or research-quality CT scans on 4 of the 6 participants in the study, and a clinical MRI scan for the remaining 2 participants. MRI images were obtained on a 1.5-T scanner at the Hospital of the University of Pennsylvania. MRI scanning parameters acquired T-1-weighted spoiled gradient-recalled-echo (MPRAGE; 35/7, 45 flip angle, one signal acquired) transverse images with 1-mm slice thickness, no gap, $1 \mathrm{~mm}$ inplane resolution. A $192 \times 256$ matrix was used throughout acquisition. CT scans were obtained using 44 contiguous axial slices covering the entire brain (2.55-mm slide thickness) without contrast.

The MRIcron image analysis program was used to identify brain lesions (www.psychology.nottingham.ac.uk/staff/crl/mricron.html). A trained research team member 
blind to the behavioral data and supervised by a neurologist segmented the lesions (for reliability details see Jax, Buxbaum \& Moll, 2006). Damage to Brodmann's area was calculated for each participant in voxels using the maps included in MRIcron which are based on templates from Damasio and Damasio (1989). (See Table 2). Brodmann's areas were subject to analysis if $\geq 10 \%$ of voxels were damaged in at least one-third of the participants. To improve power, Brodmann's areas were combined into larger regions of interest (BA $21=$ middle temporal gyrus (MTG); BA 22, $41 \& 42=$ superior temporal gyrus (STG); BA 37 = posterior temporaloccipital (TO); BA $39 \& 40=$ inferior parietal lobe (IPL); BA $44 \& 45=$ inferior frontal gyrus (IFG); BA 6 = Premotor cortex, (PM)) by calculating percent damage of total possible voxels in these regions.

\section{Experimental Procedure and Apparatus}

Before the experiment participants washed their hands with soap and water, and dried them thoroughly. Participants sat with the elbow flexed, parallel to the table with the object aligned with the shoulder. Participants were then asked to perform lifting movements with familiar objects and novel objects. After an auditory cue, participants reached forward to grasp and lift the familiar or novel object to a height in alignment with a $6 \mathrm{~cm}$ vertical marker. After holding the object briefly in the air (3 s) they returned it to the table. Participants lifted each object in a block of five trials. Series order (familiar, novel) and object order were counterbalanced across participants. Stroke participants lifted objects with the less-impaired (ipsilesional) left hand. Three control participants lifted objects with the right hand and 2 with the left hand. ${ }^{2}$

We used multiaxial force transducers (Nano17; ATI Industrial Automation, Garner, NC, USA). Each transducer measured two orthogonal force components, normal (grip) and tangential (load; .0025N resolution), during the grip-lift tasks. An electromagnetic position-angle sensor (Polhemus Fastrack, Colchester, VT, USA), secured to each object, measured vertical position (.03 in accuracy) and roll $\left(0.15^{\circ} \mathrm{RMS}\right.$ accuracy). The data was acquired using an integrated system (MotionMonitor ${ }^{\mathrm{TM}}$ Innsport Technologies, Inc.) and analyzed off-line using MATLAB 7.0. Force and position signals were sampled at $120 \mathrm{~Hz}$. Data were filtered with a second-order Butterworth filter with zero phase lag using a cut-off frequency of $8 \mathrm{~Hz}$. Load force was obtained from the normal force, and the load and grip forces at the thumb and index finger were summed and averaged.

Familiar objects were lifted off a plastic plate covering a single force transducer placed horizontally on its side on the table (see Fig. 1A). Load force was measured as vertical lift of the object was initiated and object position was documented throughout the lift. This apparatus enabled measurement of load force, object position, timing of lift-off and the derivatives of load force rate and lift acceleration. Familiar objects included a soda bottle $(649 \mathrm{~g})$, soda can (370 g), videotape (190 g), and plastic cup (55 g). A pre-experiment survey indicated that the participants had lifted each familiar object at least once a week with the lifting hand, and in the case of stroke participants, that these items were lifted both before and after the stroke (See Table 1).

Novel objects were lifted using a custom designed grip device (Fig. 1B) equipped with two parallel multi-axial force transducers. The parallel transducers had exchangeable contact surfaces $3.5 \mathrm{~cm}$ in diameter, covered with sandpaper (200 grit). The grip width or horizontal distance between the contact surfaces was $4.2 \mathrm{~cm}$. This grip device allowed for the additional

\footnotetext{
${ }^{2}$ The controls used in this study also took part in a different experiment that compared their performance to right hemisphere stroke participants who used their right hands; hence, half of the controls were run with their right hands. These data will be reported in a separate manuscript. Performance of the controls on behavioral, sensory, and force rate measures was compared with Mann-Whitney U tests based on whether they were assigned to use their right or left hand in the experiment. No differences were detected between the two groups of controls for the frequency with which they lifted the familiar objects, sensory ability, or the force rate applied (all p's non-significant).
} 
measurement of grip force and its derivatives. Three red, novel objects equal in volume were attached to the grip device (Fig. 1B) to avoid size-weight or color-weight illusions. However, the objects were different in geometric shape to assist in the development of an association between shape and weight (Gordon et al., 1991; Shick \& Plack, 1975). The novel objects included a pyramid $(400 \mathrm{~g})$, cube $(600 \mathrm{~g})$, and cylinder $(200 \mathrm{~g})$.

At the end of the experiment, participants were asked to point to each of the four familiar objects and the three novel objects separately in response to verbal queries about the order of their respective weights. The participants' ordering of objects by weight was compared to the actual weights and scored according for accuracy. The possible score for familiar objects ranged from $0-4$, and for novel objects from $0-3$.

\section{Analysis}

Temporal, force, and position measures were taken from Trial 1 and Trial 5 from each block of five trials for the novel and familiar objects. These measures were used to help define the moment of peak load or grip force, calculate force rate, identify the moment of object lift-off, and derive acceleration.

The onset of grip force and load force was denoted when the values were above a $1 \mathrm{~N}$ threshold. The grip force rate (GFR) and load force rate (LFR) were derived from the summed load and average grip forces using a 65-point numerical differentiation (i.e. calculated with a $612.5 \mathrm{~ms}$ window). Anticipatory scaling (planning) of fingertip forces was measured by the first peak LFR for familiar objects and the first peak GFR and LFR for novel objects (Johansson and Westling, 1988; Gordon et al., 1993). First peak force rates were used for subsequent statistical analyses because, in cases where there are multiple peaks in the force rate profile, the first peak occurs earlier in the lift than the maximum peak force rate. Earlier measures of force are more likely to reflect anticipatory control because they occur prior to object lift-off. Once lift-off occurs, sensorimotor feedback about object weight is available to influence the force applied. The first peak GFR and LFR was defined as the first independent peak (a peak that drops by at least $50 \%$ of its original magnitude before increasing again) above $2 \mathrm{~N} / \mathrm{s}$ that occurred before object lift-off and was associated with a positive force value.

In addition to raw force rate, we also examined derived measures in many of the statistical analyses because they provide a useful measure of the accuracy of force scaling. First, the accuracy of force output to object weight was examined by comparing the ordering of forces as a function of weight (hereafter, GFR Order and LFR Order), where values reflect the percentage of correctly ordered objects. LFR for familiar objects and GFR and LFR for novel objects were graded as correctly scaled if the force applied was greater than that used for objects of a lighter weight, less than that applied to objects of a heavier weight, and separated from other objects by at least $0.5 \mathrm{~N} / \mathrm{s}$. Each of the five control participants had a lower boundary of $0.6 \mathrm{~N} / \mathrm{s}$ of separation between objects. The percentage of correctly ordered object pairs was calculated (GFR Order and LFR Order). The second derived measure explored the sensitivity of distinguishing objects (that differ by a minimum of $200 \mathrm{~g}$ ) by measuring the inter-item spread between observed forces (hereafter, GFR Distance and LFR Distance) by taking the sum of inter-item difference scores for all possible object weight pairs. Positive Distance scores reflect the magnitude of spread between forces that are correctly scaled to object weight, negative values reflect the spread of misordered objects (i.e., a higher rate of force was used for lighter than heavier objects), and 0 indicates no difference in the forces applied to the objects. Although these derived variables still reflect the general pattern of typical GFR and LFR variables, they allow the use of statistical measures that do not simply relate one object-specific force output to another, and have been found useful in identifying subgroups of stroke participants who demonstrate subtle planning deficits (for a similar logic and method see Li et al., 2007 and Raghavan et al., 2006). 
In order to determine the relationship between anticipatory force and behavioral performance, force rate (GFR and LFR for novel objects and LFR for familiar objects) and derived force rate (Order and Distance variables) were entered into Spearman's rank correlations with GTS score, sensorimotor task performance, participant-reported frequency of lifting each familiar object, and post-experiment verbal ordering of objects by weight. The derived variables were further examined in association with damaged brain regions, and significant results were subjected to hierarchical regressions to determine whether total lesion volume contributed to the prediction of force rate variables.

Force rate variables were also compared against object weights using an ordinal nonparametric measure of association, Somers' D (Kendall \& Gibbons, 1990), a modified form of a gamma test. Unlike gamma, which does not correct for ties between pairs of ranked data, Somers' D penalizes for tied pairs only when they occur in the predicted variable, in this case, force rate scaling, rather than the predictor, object weight. This treatment of ties reflects the directional hypothesis that object weight predicts force rate scaling. Somers' D reaches a maximum score of 1.0 and a minimum score of -1.0 for perfectly ordered monotonic associations, where 0 reflects independence.

Between-group differences were further tested in the LFR Distance measure on Trial 1 with familiar objects using an unequal variances t-test. An unequal variances test was used because the data failed a Levene's test for the equality of variance $(F=6.10, p=.034)$, indicating that the shape of the distributions was different between controls and patients. However, the data was still normally distributed, as determined by a Shapiro-Wilk test for normality ( $\mathrm{W}=.98, \mathrm{p}$ $=.94$, patients; $\mathrm{W}=.87, \mathrm{p}=.26$, controls). We then used Siegel-Tukey tests (Siegel \& Tukey, $1960)$ to determine whether within-group variability was greater in the patient than the control group for the LFR Distance measure on Trial 1 with familiar objects. The null hypothesis of the Siegel-Tukey test is that for two groups of observations arranged in ascending order, the observations from each group will be randomly mixed among the ranks of high and low scores.

Because in most cases the measures were related to one another, a Keppel's Modified Bonferroni correction (Keppel, 1991) was used set the critical p-value for significance to reduce the likelihood of committing a Type II error. Statistical evaluation of all group differences was determined using two-tailed exact p-values where appropriate.

\section{RESULTS}

\section{GFR and LFR Traces}

Participants with IMA did not show scaling of GFR or LFR on the first trial with familiar objects, but did by the 5 th trial. Figure 2 shows representative force traces for the first trial lifting familiar objects. The controls and LCVA exhibited initial scaling which improved in differentiation by the $5^{\text {th }}$ trial.

Between Groups Comparison of the Scaling of Force to Object Weight-To assess whether force rate monotonically scales with object weight, where heavier objects elicit higher force rates, we used a series of Somers' D measures of association. For the familiar objects, the association of LFR to object weight was much lower on Trial 1 than that found on Trial 5 in the patients (Trial 1, $\mathrm{d}=.49$; Trial 5, $\mathrm{d}=.68$; see Figure $3 \mathrm{~B}$ ) but not in controls (Trial 1, $\mathrm{d}=$. 81; Trial 5, d = .80; see Figure 3A). For novel objects, both patients and controls showed better concordance in LFR than GFR with the weight of the novel objects and concordance improved in both LFR and GFR from Trial 1 to 5 (Control: LFR Trial 1, d = .41; LFR Trial 5, d = .81; GFR Trial 1, $\mathrm{d}=.20$; GFR Trial 5, $\mathrm{d}=.41$, see Figure 3C and 3D), however, the patient group had poorer concordance across all measures (Control: LFR Trial 1, $\mathrm{d}=.15$; LFR Trial 5, $\mathrm{d}=$. 37; GFR Trial 1, $\mathrm{d}=.57$; GFR Trial 5, $\mathrm{d}=.63$; see Figure 3E and 3F). 
In a between-groups analysis, there was no significant difference between patients and controls in LFR Distance measure on Trial 1 with familiar objects, $\mathrm{t}(6)=-.45, \mathrm{p}=.67$, where the median and quartiles in patients and controls was $12.22(10.2,43.3)$ and $11.72(23.9,37.8)$,

respectively. Despite the lack of a significant difference in LFR Distance between patients and controls, the controls had more widely dispersed LFR Distance values as compared to patients $(\mathrm{p}=.052$, Siegel-Tukey test). This indicates that patients applied less distinct force rates based on the identity of the objects (see also Figure 3A and B).

To assess for temporal differences in force loading, between-group differences in peak acceleration for each object were compared in a Mann-Whitney U. An examination of peak acceleration for each object revealed no statistically significant difference between the patients and control participants (all U's > 9.0, $\mathrm{n}_{1}=6 \mathrm{n}_{2}=5$, all p's $>.355$ ). The overall medians and quartiles in patients and controls were $193.0 \mathrm{~cm} / \mathrm{s}^{2}$ [163, 236] and $200.2 \mathrm{~cm} / \mathrm{s}^{2}[113,241]$, respectively. The similarity of peak acceleration suggests that patients were no different than controls in terms of how fast their speed changed at the moment when load and grip force were measured.

\section{Derived Variables}

Sensory Deficits and Frequency of Lifting Familiar Objects-The relationships between the derived force rate variables and the clinical data listed in Table 1 were evaluated using Spearman's correlations. There were no significant correlations between force rate and the clinical data (all $\mathrm{r}_{\mathrm{s}}$ 's $<.50$, all $\mathrm{p}$-values $>.116$, uncorrected for the 64 comparisons performed), however, based on the small sample size, this null result should be viewed with caution. We examined the correlation between LFR and GFR for each object and the derived scores (Order and Distance) on Trial 1 and 5 against the participant-reported frequency of lifting for each familiar object with the lifting hand. Higher frequency of lifting a soda bottle during everyday life correlated with better LFR Distance on Trial $5\left(\mathrm{r}_{\mathrm{s}}=.72, \mathrm{p}=.012\right)$.

Correlations for other objects were not significant in this sample.

The force rate and derived score for each familiar object on Trial 1 and 5 were correlated against the post-experiment verbal ordering of objects by weight. Controls and participants with LCVA were equally capable of explicitly ordering the 4 familiar objects, Median $=4$ for both control and LCVA. They were both equally poor at explicitly ordering the 3 novel objects, Median=1. We were unable to find significant correlations between the accuracy of verbal ordering by object weight (familiar and novel) and the derived force rate measures (all p-values > .05). Again, these null results must be viewed with caution.

Influence of Ideomotor Apraxia-We examined the relationship between the GTS test of IMA and the derived GFR and LFR measures (see Table 3). As can be seen in Figure 4, there was a significant correlation between the ability to predict the weight of familiar objects, as measured by LFR Distance on Trial 1 , and the GTS test of IMA $\left(\mathrm{r}_{\mathrm{s}}=.820, \mathrm{p}=.046\right)$. GFR and LFR Distance did not correlate with GTS on other trials, or with novel objects (all $\mathrm{r}_{\mathrm{s}}$ 's $<.395$, all p's $>$.230). These data indicate, consistent with qualitative observations of the GFR and LFR data, that there is a relationship between the ability to correctly scale fingertip forces for lifts of familiar objects and IMA.

Brain Lesion Analysis-The relationship between the GFR and LFR derived variables and damaged brain regions was examined in separate Spearman's rank correlations. As shown in Table 4 and Figure 5 and Figure 6, the STG, MTG and IPL were significantly associated with poor load force rate scaling ( $L F R$ Order) during lifts of familiar objects on Trial $1 .^{3}$ The remaining GFR and LFR Order and Distance variables for familiar and novel objects were not significantly correlated with damage to any brain region of interest (p-values $>.09$ ). 
To assess whether total lesion volume contributed to the observed significant correlation between LFR Order and STG and IPL damage, a hierarchical regression was performed ${ }^{4}$. $L F R$ Order on Trial 1 with familiar objects was entered as the dependent measure and voxel count measures of total lesion volume as well as STG, MTG or IPL damage were entered as predictors. Because STG, MTG and IPL were assumed to predict the most variance in a regression, they were each entered last in their respective regressions. The regression model of STG alone yielded the best fit, with $94.6 \%$ of the variance explained, $R=.97, F(1,4)=69.93$, $\mathrm{p}=.001$, and excluded the predictors of total lesion volume, Beta In $=.12, \mathrm{p}=.61$, IPL, Beta $\mathrm{In}=-.01, \mathrm{p}=.97$, and MTG, Beta $\mathrm{In}=.32, \mathrm{p}=.34$. A regression model with total lesion volume alone, $\mathrm{F}(1,4)=3.12$, $\mathrm{p}=.15$, was a poor fit.

\section{DISCUSSION}

In this study we sought to determine whether the memories that underlie anticipatory fingertip force control when lifting objects are based on object-specific representations, or whether object density (and thus the force required) is estimated based on the immediate visual assessment of an object's structural properties (Cole, 2008). We investigated this issue by comparing anticipatory force scaling during lifts of familiar objects to lifts of novel objects with equivalent volumes but varying weight. Performance differences observed during lifts of familiar versus novel objects suggest that different mechanisms contribute to the ability to scale fingertip forces. Based on a two-route model of action, we predicted that this would be the case. We assessed this prediction in patients with IMA, who are known to have deficits in planning actions with familiar objects (Johnson-Frey, 2004; Glover, 2004; Buxbaum et al., 2003; Sirigu et al., 1995), even in the ipsilesional hand. We also assessed the prediction that lesions to the ventro-dorsal stream (IPL and STG) would disrupt production of anticipatory grip and load force during lifts of familiar objects.

The study predictions were confirmed in a preliminary sample of 6 stroke patients. Examination of individual participant's force traces revealed poor anticipatory control with familiar objects (Trial 1 LFR) in those with IMA. In between-group analyses, patients did not show anticipatory scaling of load force rate to familiar object weight. However, they improved in their scaling by Trial 5 with both familiar and novel objects. On Trial 1, patients seemed to generate a more tightly clustered set of load force rates (that did not distinguish as well between the familiar object weights) than controls. We also found that Trial 1 LFR for familiar objects correlated significantly with our measure of IMA, Gesture to Sight of objects, and with lesions to the inferior parietal lobe and the posterior portion of the middle and superior temporal cortices, but not with overall lesion volume. These data suggest that the ipsilesional deficits previously observed in IMA patients with left temporal-parietal lesions in the planning of grip posture with familiar objects (Buxbaum, Johnson-Frey \& Bartlett-Williams, 2005) may extend to the planning of fingertip forces during object lifts. While we did not initially predict the involvement of posterior MTG in anticipatory grip force, this area consistently shows heightened activity in imaging studies when participants view manipulable objects and tools (Chao, Haxby \& Martin, 1999; Moore \& Price, 1999; Beauchamp, 2002), and MTG has been cited as part of the network involved in conceptual object representations. Thus, it is possible that information required for force scaling is among the attributes represented by this network. Future studies will be required to explore this possibility.

\footnotetext{
${ }^{3}$ Performance on Trial 5 with novel objects was at "ceiling" (maximum possible score) for most participants, and thus did not demonstrate enough variability to be entered into a correlation with brain regions of interest.

${ }^{4}$ Pearson's correlations showed that the STG was significantly correlated with total lesion volume, $r=0.86, p=.028$ and the IPL was near significant, $r=0.80, p=.056$. On inspection of the Variable Inflation Factors they were found to be below 4, indicating that multicollinearity is low (Myers, 1990), and thus it was acceptable to enter each variable as a predictor with total lesion volume into individual regressions.
} 
The poor performance of IMA participants when interacting with familiar objects stands in contrast to their performance during lifts of novel objects, which resembled controls even on early trials. None of the force measures relevant to lifts of novel objects correlated with IMA, nor were these measures affected by the integrity of any particular brain regions, at least in this small sample. These data suggest that the procedures and neural substrates for planning of force production for familiar as compared to novel objects may be at least partly distinct.

An additional notable aspect of the data is that after repeated exposures (i.e., by Trial 5), the performance of the IMA participants during lifts of familiar objects was indistinguishable from controls. This raises the possibility that appropriate interim internal models used for anticipatory force scaling may improve or develop over the short-term. Unfortunately, we did not require our participants to return for testing of retention on subsequent days, thus, the longevity of these newly-trained representations is unclear. Given its potential relevance to rehabilitation, this question warrants further investigation because of its potential role as a rehabilitative strategy.

The present data may be viewed as a complement to a recent report by Li and colleagues (2007) of an IMA patient who demonstrated impaired anticipatory control on the first trial during lifts of novel objects when those objects violated typical size-weight associations. The authors suggested that the patient did not seem to rely upon stored information to anticipate expected weight. Instead, the patient appeared to use recent sensorimotor memory to guide force production, resulting in a grip force that was appropriate to the previous (and not current) trial. Consistent with this possibility, Gordon et al. (1991) suggested that different procedures may be used for anticipatory control based on short-term sensorimotor memories as opposed to long-term, established representations of object properties. Like our patients, the patient in Li et al.'s study (2007) also demonstrated normal GF learning over time. A disparity between deficient use of remotely-acquired information and the intact ability to learn over the short term is consistent across both studies. This suggests that the representational substrates of remote and short-term memories for force control are an additional area meriting further investigation. Thus far it has been established that associative and sensorimotor memories formed while lifting objects remain in healthy subjects for at least 24 hours (Nowak, Koupan \& Hermsdörfer, 2007; Gordon, Forssberg, \& Iwasaki, 1994). However, in cases where there is damage to the IPL and/or STG, one might observe sufficient learning over the short-term, coupled with an inability to develop long-term internal models.

In summary, the present study supports the hypothesis that the ventro-dorsal system of the left hemisphere, in addition to its known specialization for control of hand posture and planning of skilled actions, could also be involved in anticipatory scaling of fingertip forces during lifts of familiar objects. Given that our LCVA patients could explicitly order familiar objects by weight prior to lifting them, the data suggest that lesions to the left temporoparietal area do not affect weight knowledge per se, but rather the ability to integrate object property information into an internal model of the dynamics of hand-object interaction (Wolpert \& Flanagan, 2001; Kawato et al., 2003; Flanagan \& Tresilian, 1994). One caveat is that the results are based on a small sample of patients which may not have been sufficient to detect some differences. For example, while we did not find any relationship between our force measures and clinical measures of hand function, other studies have found a consistent relationship (for example, see Gordon \& Duff, 1999). However, both behavioral and neuroanatomic data are consistent with our theoretically-based predictions, and suggest that replication with a larger sample of patients will be of interest. Such a study is currently underway in our lab.

By relating predictions relevant to force scaling to the ventro-dorsal stream several implications emerge for memory durability and interlimb transfer that are relevant to the rehabilitation of anticipatory fingertip force control. Differential predictions based on lesion location could be 
used to plan effective rehabilitation programs for patients and may have implications for recent efforts in adult stroke patients to train the contralesional limb through interlimb transfer tasks. During interlimb transfer training novel objects are lifted with the ipsilesional limb first then the contralesional limb. Previous studies have consistently tested only immediate or nearimmediate transfer effects (Gordon, 1994; Raghavan et al., 2006; Chang, Flanagan \& Goodale, 2008). However, one might postulate that patients with IPL and temporal lobe damage are capable only of short-lived sensorimotor learning, and would not be able to consolidate that information into stored knowledge about an object. The current study is consistent with the idea that rehabilitation efforts, including interlimb transfer training, should be tailored to take into account the area of damage and checked at long delays to establish that newly formed representations are durable enough to be relevant to rehabilitation goals.

\section{Acknowledgments}

This project is funded, in part, under a grant with the Pennsylvania Department of Health. The Department specifically disclaims responsibility for any analyses, interpretations, or conclusions. This study was funded by the Albert Einstein Society, Albert Einstein Healthcare Network, Philadelphia, PA, and by NIH grant NS036387 to Laurel Buxbaum. We thank Dan Kimberg, Branch Coslett, and Steve Jax for assistance with lesion segmentation and analyses, Marcus Besserand Paul Nagelkerke for technical support, and Binny Talatiand Jeff Collins for assistance with data collection.

\section{REFERENCES}

Beauchamp MS, Lee KSE, Haxby JV, Martin A. Parallel visual motion processing streams for manipulable objects and human movements. Neuron 2002;34:149-159. [PubMed: 11931749]

Bechtol CO. The use of a dynamometer with adjustable handle spacings. J Bone Jt Surg 1954;36A:820824.

Blennerhassett JM, Carey LM, Matyas TA. Grip force regulation during pinch grip lifts under somatosensory guidance: Comparison between people with stroke and healthy controls. Arch Phys Med Rehabil 2006;87:418-429. [PubMed: 16500179]

Boecker H, Lee A, Mühlau M, Ceballos-Baumann AO, Ritzl A, Spilker M, Marquardt C, Hermsdörfer J. Force level independent representation of predictive grip force-load force coupling: a PET activation study. Neuroimage 2005;25:243-252. [PubMed: 15734359]

Buxbaum LJ. Ideomotor apraxia: a call to action. Neurocase 2001;7:445-458. [PubMed: 11788737]

Buxbaum LJ, Frey SH, Bartlett-Williams M. Deficient internal models for planning hand-object interactions in apraxia. Neuropsychologia 2005;43:917-929. [PubMed: 15716162]

Buxbaum LJ, Kyle KM, Grossman M, Coslett HB. Left inferior parietal representations for skilled handobject interactions: Evidence from stroke and corticobasal degeneration. Cortex 2007;43:411-423. [PubMed: 17533764]

Buxbaum LJ, Kyle K, Menon R. On beyond mirror neurons: Internal representations subserving imitation and recognition of skilled object-related actions in humans. Cogn Brain Res 2005;25:226-239.

Buxbaum LJ, Sirigu A, Schwartz MF, Klatzky R. Cognitive representations of hand posture in ideomotor apraxia. Neuropsychologia 2003;41:1091-1113. [PubMed: 12667544]

Chang EC, Flanagan JR, Goodale MA. The intermanual transfer of anticipatory force control in precision grip lifting is not influenced by the perception of weight. Exp Brain Res 2008;185:319-329. [PubMed: 17934725]

Chao LL, Haxby JV, Martin A. Attribute-based neural substrates in posterior temporal cortex for perceiving and knowing about objects. Nat Neurosci 1999;2:913-919. [PubMed: 10491613]

Cole KJ. Lifting a familiar object: Visual size analysis, not memory for object weight, scales lift force. Exp Brain Res 2008;188:551-557. [PubMed: 18443767]

Creem-Regehr SH, Lee JN. Neural representations of graspable objects: Are tools special? Cogn Brain Res 2005;22:457-469.

Dafotakis M, Grefkes C, Eickhoff SB, Karbe H, Fink GR, Nowak DA. Effects of rTMS on grip force control following subcortical stroke. Exp Neurol 2008;2211:407-412. [PubMed: 18395715]

Damasio, H.; Damasio, AR. Lesion Analysis in Neuropsychology. New York: Oxford; 1989. 
Dellon AL. The plastic ridge device and moving two-point discrimination. J Hand Surg Am 1980;5:9293. [PubMed: 7365223]

Duff SV, Gordon AM. Learning of grasp control in children with hemiplegic cerebral palsy. Dev Med Child Neurol 2003;45:746-757. [PubMed: 14580130]

Ehrsson HH, Fagergren A, Forssberg H. Differential fronto-parietal activation depending on the force used in a precision grip task: an fMRI Study. J Neurophysiol 2001;85:2613-2623. [PubMed: 11387405]

Flanagan JR, Beltzner MA. Independence of perceptual and sensorimotor predictions in the size-weight illusion. Nat Neurosci 2000;3:737-741. [PubMed: 10862708]

Flanagan JR, Tresilian JR. Grip-load force coupling: a general control strategy for transporting objects. Journal J Exp Psychol Hum Percept Perform 1994;20:677-685.

Frey SH. What puts the how in where? Tool use and the divided visual streams hypothesis. Cortex 2007;43:368-375. [PubMed: 17533760]

Glover S. Separate visual representations in the planning and control of action. Behav Brain Sci 2004;27:3-24. [PubMed: 15481943]

Gordon AM, Duff SV. Relation between clinical measures and fine manipulative control in children with hemiplegic cerebral palsy. Dev Med Child Neurol 1999;41:586-591. [PubMed: 10503916]

Gordon A, Forssberg H, Iwasaki N. Formation and lateralization of internal representations underlying motor commands during precision grip. Neuropsychologia 1994;32:555-568. [PubMed: 8084414]

Gordon AM, Forssberg H, Johansson RS, Westling G. Integration of sensory information during the programming of precision grip: Comments on the contributions of size cues. Exp Brain Res 1991;85:226-229. [PubMed: 1884761]

Gordon AM, Westling G, Cole KJ, Johansson RS. Memory representations underlying motor commands used during manipulation of common and novel objects. J Neurophysiol 1993;69:1789-1796. [PubMed: 8350123]

Grandy MS, Westwood DA. Opposite perceptual and sensorimotor responses to a size-weight illusion. J Neurophysiol 2006;95:3887-3892. [PubMed: 16641383]

Haaland KY, Harrington DL, Knight RT. Spatial deficits in ideomotor limb apraxia: a kinematic analysis of aiming movements. Brain 1999;122:1169-1182. [PubMed: 10356068]

Jax SA, Buxbaum LJ, Moll AD. Deficits in movement planning and intrinsic coordinate control in ideomotor apraxia. J Cogn Neurosci 2006;18:2063-2076. [PubMed: 17129191]

Johnson-Frey SH. The neural bases of complex human tool use. Trends Cogn. Sci 2004;8:71-78. [PubMed: 15588811]

Kawato M, Kuroda T, Imamizu H, Nakano E, Miyauchi S, Yoshioka T. Internal forward models in the cerebellum: fMRI study on grip force and load force coupling. Prog Brain Res 2003;142:171-188. [PubMed: 12693261]

Kendall, MG.; Gibbons, JD. Rank Correlation Methods. 5th ed.. London: Arnold; 1990.

Keppel, G. Design and Analysis: A Researcher's Handbook. New York: Prentice-Hall; 1991. p. 169

Kuhtz-Buschbeck JP, Ehrsson HH, Forssberg H. Human brain activity in the control of fine static precision grip forces: an fMRI study. Eur J Neurosci 2001;14:382-390. [PubMed: 11553288]

Lewis JW. Cortical networks related to human use of tools. Neuroscientist 2006;12:211-231. [PubMed: 16684967]

Li Y, Randerath J, Goldenberg G, Hermsdörfer J. Grip forces isolated from knowledge about object properties following a left parietal lesion. Neurosci Lett 2007;426:187-191. [PubMed: 17904743]

Majdandzic J, Grol MJ, Schie HT, van Verhagen L, Toni I, Bekkering H. The role of immediate and final goals in action planning: an fMRI study. Neuroimage 2007;37:589-598. [PubMed: 17587600]

Mathiowetz V, Kashman N, Volland G, Weber K, Dowe M, Rogers S. Grip and pinch strength: normative data for adults. Arch Phys Med Rehabil 1985;66:69-72. [PubMed: 3970660]

Mathiowetz, V.; Volland, G.; Kashman, N.; Weber, K. Nine hole peg test. In: Wade, DT., editor. Measurement in Neurological Rehabilitation. New York: Oxford University Press; 1992. p. 171

Moberg E. Objective methods for determining the functional value of sensibility in the hand. J Bone Joint Surg 1958;40-B:454-476. 
Mon-Williams M, Murray AH. The size of the visual size cue used for programming manipulative forces during precision grip. Exp Brain Res 2000;135:405-410. [PubMed: 11146818]

Moore CJ, Price CJ. A functional neuroimaging study of the variables that generate category-specific object processing differences. Brain 1999;122:943-962. [PubMed: 10355678]

Myers, R. Classical and Modern Regression with Applications. 2nd ed.. Boston, MA: Duxbury Press; 1990.

Nowak DA, Grefkes C, Dafotakis M, Küst J, Karbe H, Fink GR. Dexterity is impaired at both hands following unilateral subcortical middle cerebral artery stroke. Eur J Neurosci 2007;25:3173-3184. [PubMed: 17561831]

Nowak DA, Hermsdörfer J, Topka H. Deficits of predictive grip force control during object manipulation in acute stroke. J Neurol 2003;250:850-860. [PubMed: 12883929]

Nowak DA, Koupan C, Hermsdörfer J. Formation and decay of sensorimotor and associative memory in object lifting. Eur J Appl Physiol 2007;100:719-726. [PubMed: 17503069]

Nowak DA, Timmann D, Hermsdörfer J. Dexterity in cerebellar agenesis. Neuropsychologia 2007;45:696-703. [PubMed: 16979674]

Quaney BM, Perera S, Maletsky R, Luchies CW, Nudo RJ. Impaired grip force modulation in the ipsilesional hand after unilateral middle cerebral artery stroke. Neurorehabil Neural Repair 2005;19:338-349. [PubMed: 16263966]

Quaney BM, Rotella DL, Peterson C, Cole KJ. 'Sensorimotor memory' for fingertip forces: Evidence for a task-independent motor memory. J Neurosci 2003;23:1981-1986. [PubMed: 12629204]

Pinkerton E, Humphrey NK. The apparent heaviness of colours. Nature 1975;250:164-165. [PubMed: 4843139]

Raghavan P, Krakauer JW, Gordon AM. Impaired anticipatory control of fingertip forces in patients with a pure motor or sensorimotor lacunar syndrome. Brain 2006;129:1415-1425. [PubMed: 16597653]

Rizzolatti G, Matelli M. Two different streams form the dorsal visual system: anatomy and functions. Exp Brain Res 2003;153:146-157. [PubMed: 14610633]

Schmitz C, Jenmalm P, Ehrsson HH, Forssberg H. Brain activity during predictable and unpredictable weight changes when lifting objects. J Neurophysiol 2005;93:1498-1509. [PubMed: 15385599]

Semmes, J.; Weinstein, S.; Ghent, L.; Teuber, HL. Somatosensory Changes After Penetrating Brain Wounds in Man. Cambridge, MA: Harvard University Press; 1960.

Siegel S, Tukey JW. A nonparametric sum of ranks procedure for relative spread in unpaired samples. Journal of the American Statistical Association 1960;55:429-445.

Sirigu A, Cohen L, Duhamel JR, Pillon B, Dubois B, Agid Y. A selective impairment of hand posture for object utilization in apraxia. Cortex 1995;31:41-55. [PubMed: 7781320]

Stone, JH. Sensibility. In: Casanova, JS., editor. Clinical Assessment Recommendations. Chicago, IL: American Society of Hand Therapists; 1992. p. 71-84.

Weber EH. Ueber den Tastsinn. Müller Archiv 1935:152-159.

Wetter S, Poole JL, Haaland KY. Functional implications of ipsilesional motor deficits after unilateral stroke. Arch Phys Med Rehabil 2005;86:776-781. [PubMed: 15827931]

Wolpert DM, Flanagan JR. Motor Prediction. Curr Biol 2001;18:R729-R732. [PubMed: 11566114] 


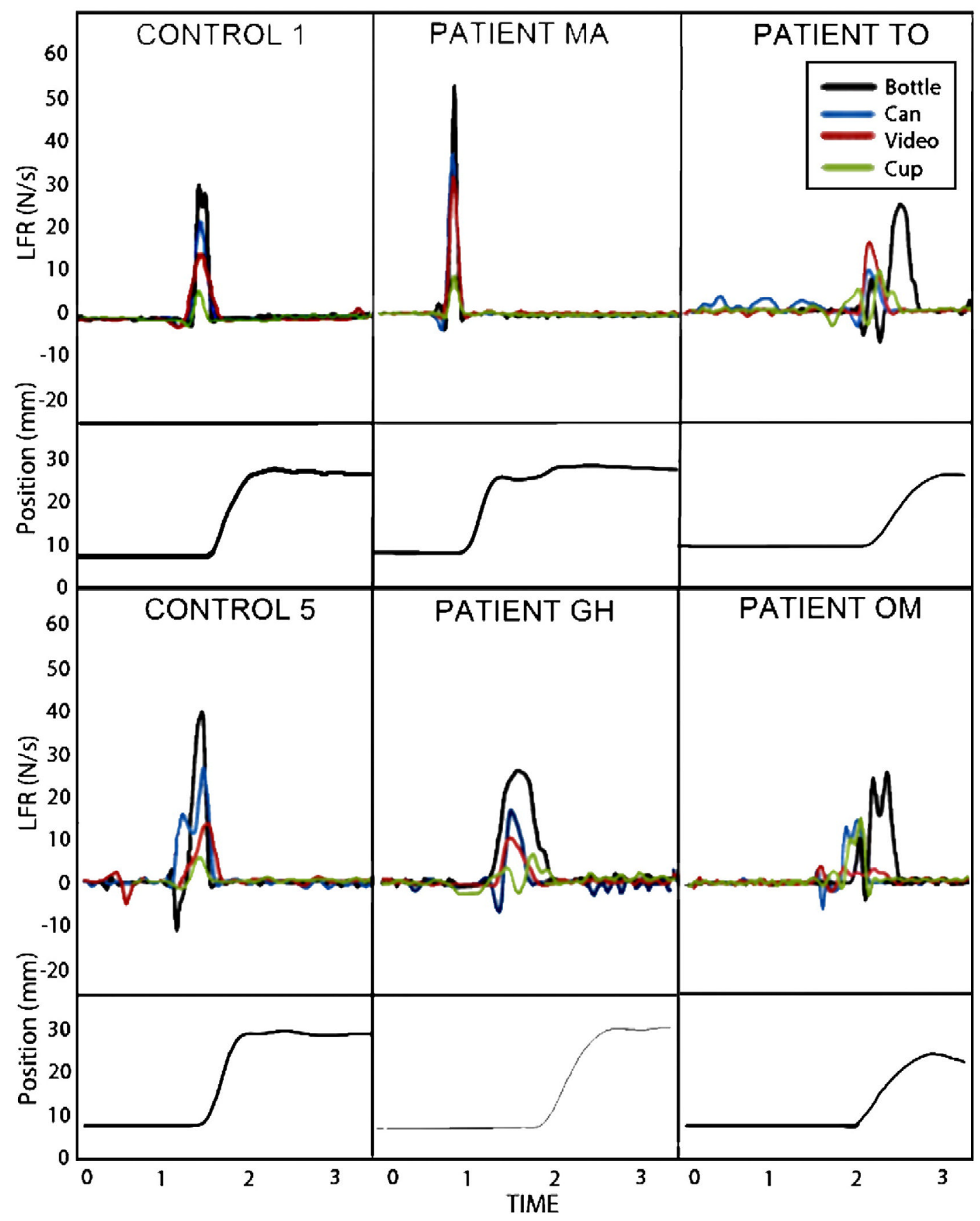

Fig. 1.

Load force rate (LFR) traces across time for Trial 1 with familiar objects for two controls, two LCVA participants without ideomotor apraxia (IMA), and two LCVA participants with IMA. Below each LFR trace is a depiction of object position to indicate the point at which object lift-off was achieved. Controls and LCVA appropriately scaled LFR based on object weight for familiar objects wherein heavier objects elicited higher LFRs. Furthermore, LFRs were primarily single-peaked and the maximumpeak value was achieved before the object is lifted off the table, indicating a smooth application of load force that precedes and anticipates sensory feedback. In contrast, IMA participants had poorly differentiated and incorrectly scaled LFRs based on object weight. They tended to generate multi-peaked waveforms with a low first peak 
before object lift-off and a second maximum peak after lift-off once sensory feedback regarding object weight was available. Other force fluctuations were noticeable in the IMA participants, including large negative load forces prior to lift-off, suggesting an attempt to stabilize the grasp, and multiple sub-peaks while holding the object aloft, indicating difficulty maintaining the grip. 

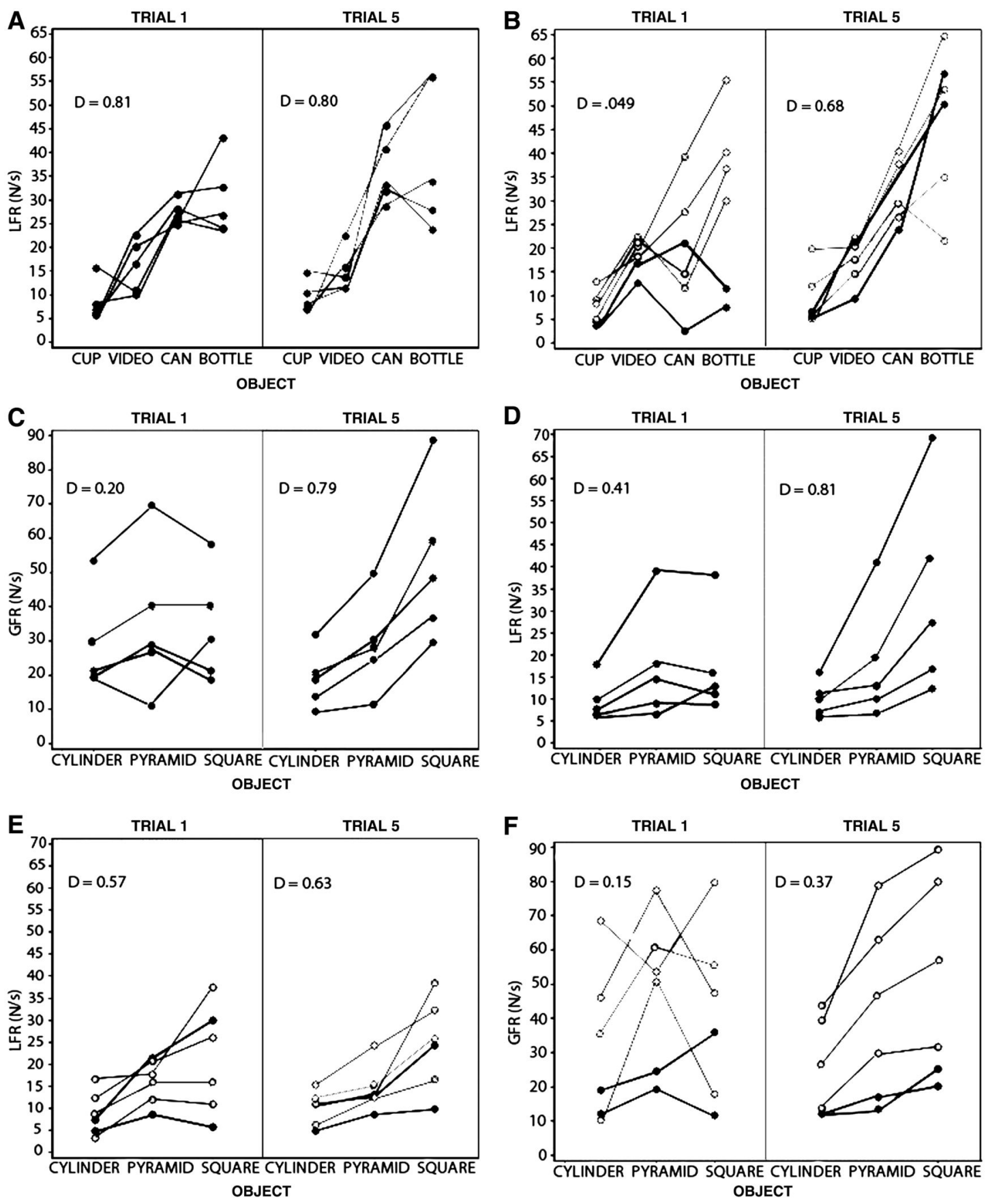

Fig 2.

Somers' Dmeasure of association between real object weight and fingertip force. Each participant's force rate is plotted by object and objects are ordered by ascending weight from left to right along the x-axis. Given that the x-values are ordered, Somers' D is the difference between concordant and discordant pairs, divided by the number of concordant, discordant and tied pairs for the X-value. (A) Control LFR for familiar objects on Trials 1 and 5. (B) Patient LFR for familiar objects on Trials 1 and 5. Apraxics are demarcated by dark filled circles and non-apraxics by open circles. (C) Control GFR for novel objects on Trials 1 and 5. (D) Control LFR for novel objects on Trials 1 and 5. (E) Patient GFR for novel objects on Trials 1 and 5.

(F) Control LFR for novel objects on Trials 1 and 5. 


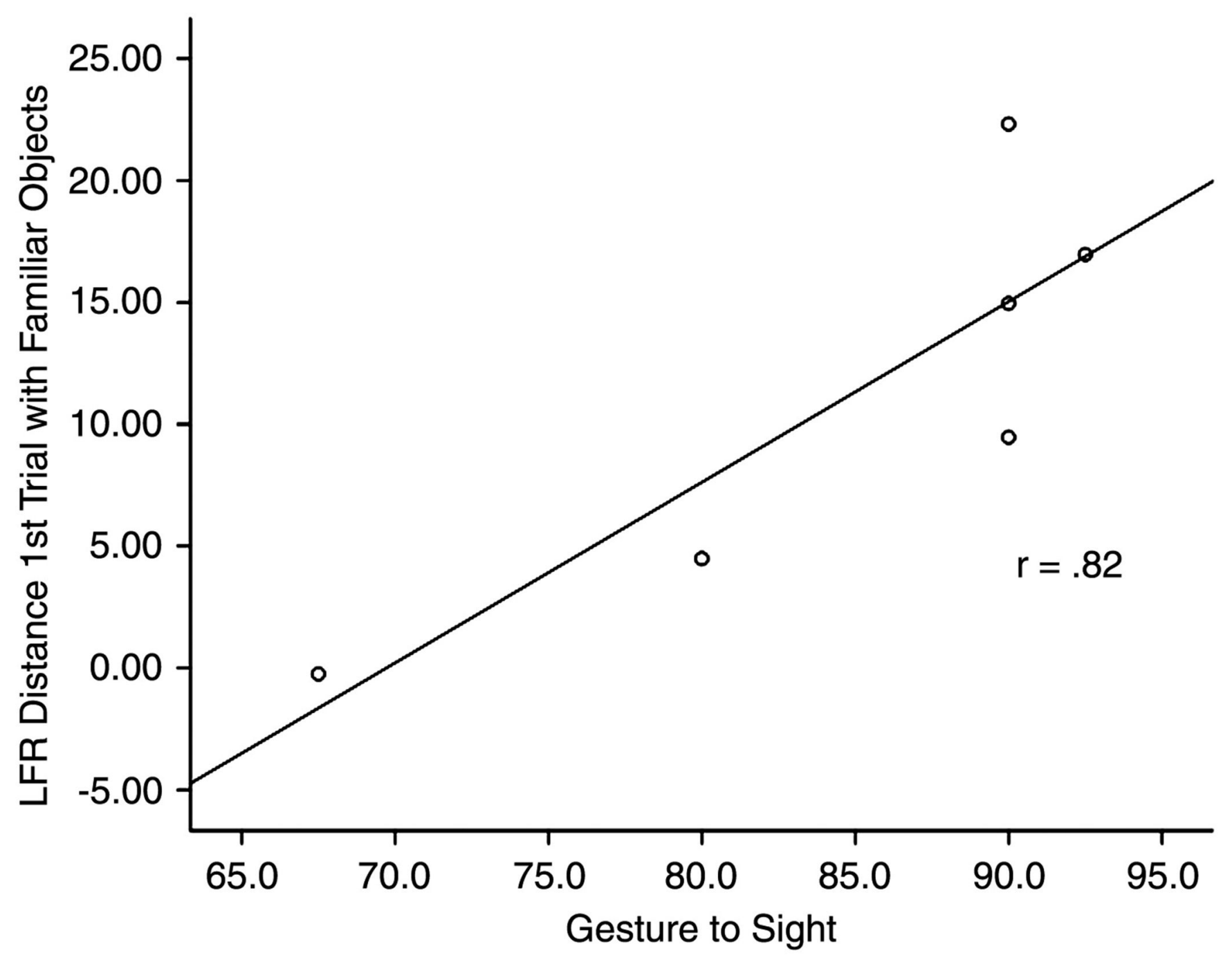

Fig. 3.

A scatterplot and regression line showing the relationship between load force rate ( $L F R)$ Distance for Trial 1 with familiar objects and the gesture to sight (GTS) test of ideomotor apraxia. $\mathrm{R}$-sq. $=0.67$ 


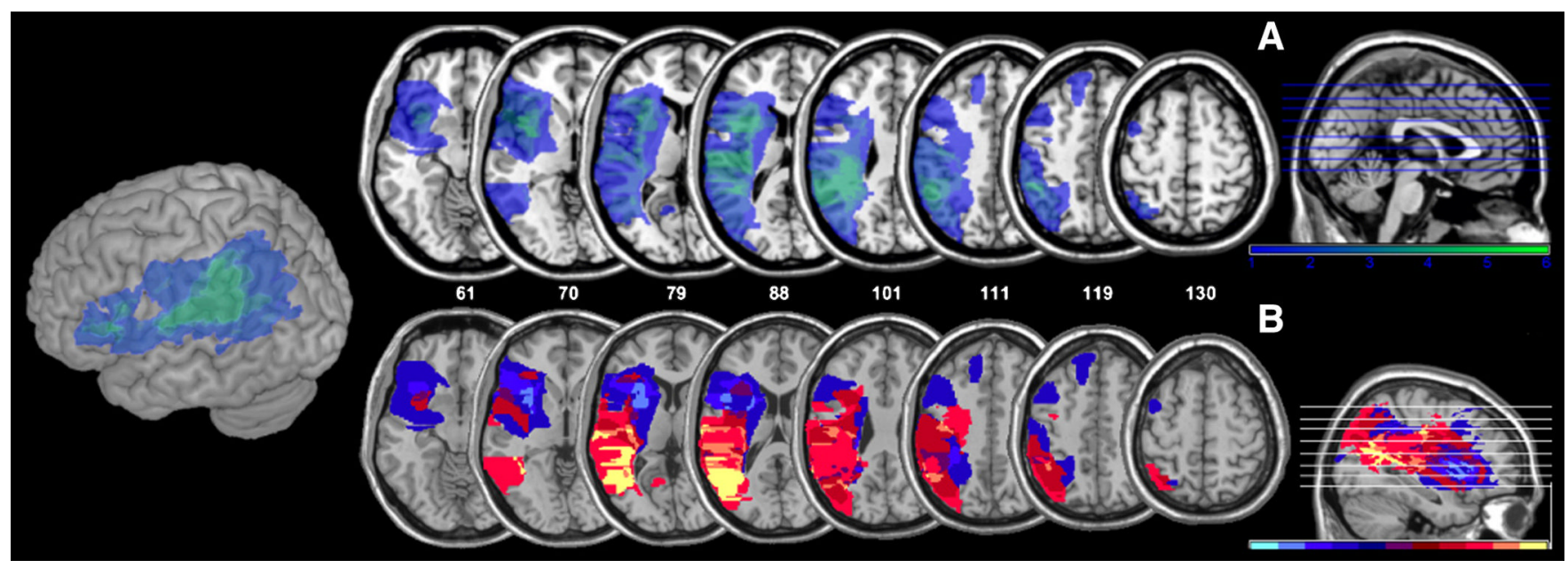

Fig. 4.

(A) Overlay maps of all patients lesions $(n=6)$ on axial slices (slice location indicated by $z-$ coordinate) and a 3D cortical rendering of the left hemisphere. Colors ranging from dark blue to light green indicate the number of patients who share the same lesion location, where the darkest color indicates lesions in only one patient and the lightest color indicates lesions in all six patients. (B) Subtraction maps of lesions of most impaired $(n=3)$ versus least impaired $(n=3)$ stroke participants based on scores on load force rate $(L F R)$ Distance Trial 1 with familiar Objects. Colors ranging from yellow to red indicate lesion loci damaged in more "most impaired" than "least impaired" participants (yellow=damaged in 3 "most impaired" and 0 "least impaired"; posterior superior temporal and inferior parietal lobes). Colors ranging from light blue to dark blue indicate regions more frequently damaged in "least impaired" than "most impaired" participants. Purple indicates regions that did not distinguish between groups (difference of 0 ). 

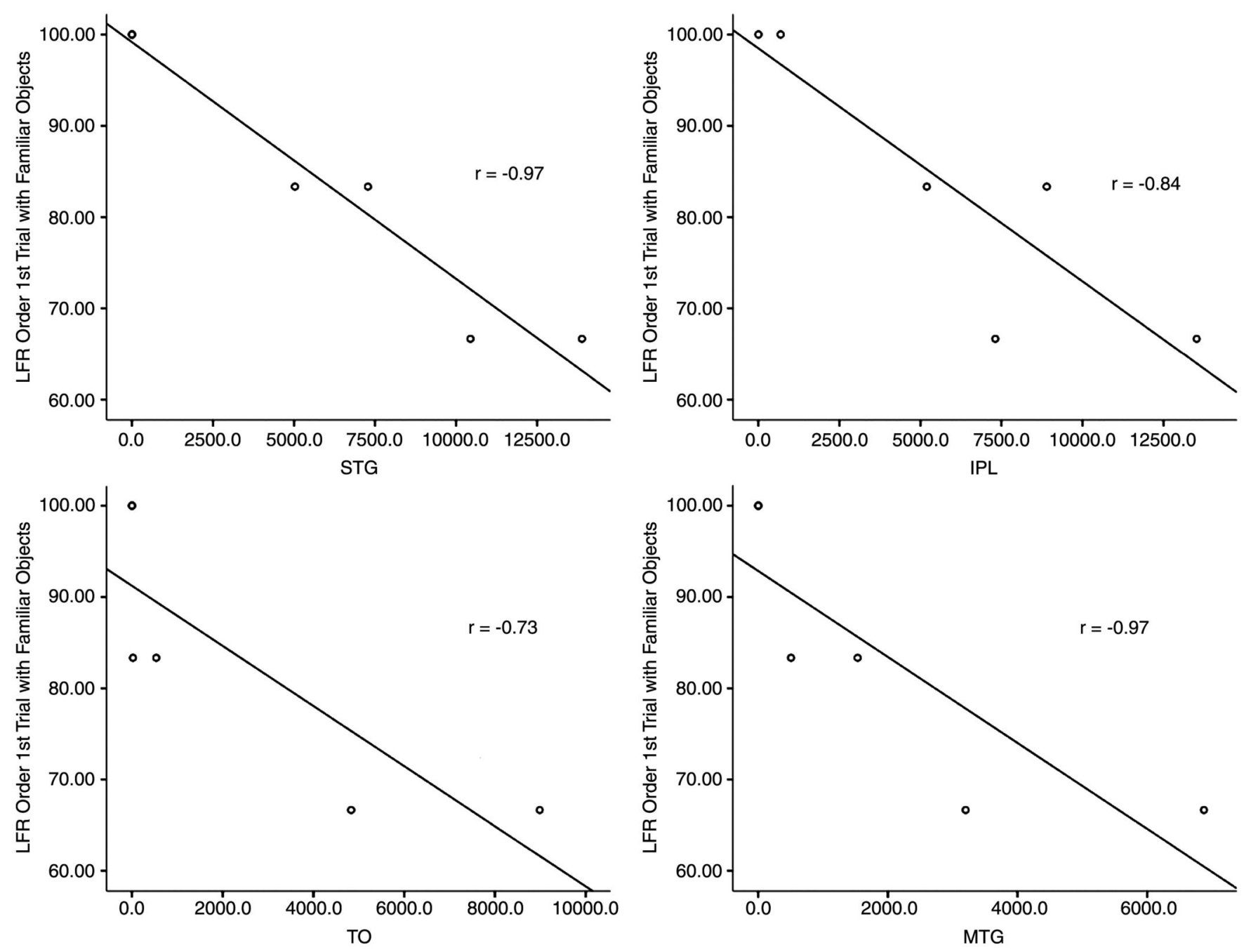

Fig. 5.

Scatterplots and regression lines showing the relationship between load force rate (LFR)

Order Trial 1 for familiar objects and number of voxels damaged in brain regions of interest.

$\mathrm{STG}=$ superior temporal gyrus; IPL=inferior parietal lobe; $\mathrm{TO}=$ posterior temporal-occipital; MTG=middle temporal gyrus. 

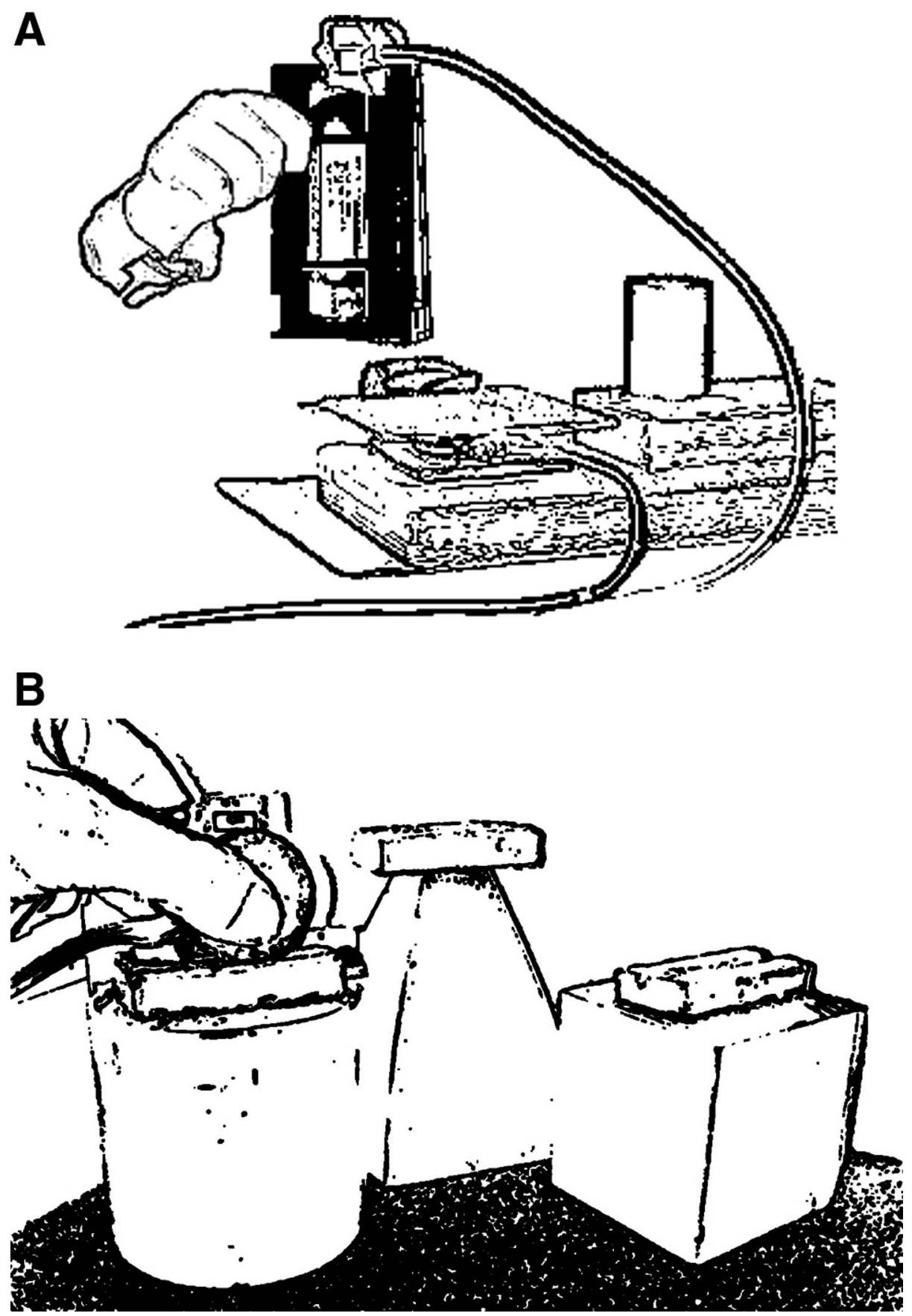

Fig. 6.

Experimental setup. (A) Familiar object: In this case, videotape, with a Polhemus position sensor (Polhemus Fastrack, Colchester, VT) attached being lifted from a platform attached to a 6 DOF force transducer (Nano17; ATI Industrial Automation, Garner, NC, USA), placed horizontally, acting as a load cell. (B) Novel objects: One of three geometric shaped objects (Cube shown) attached to a grip device consisting of two parallel 6 DOF force transducers with contact surfaces made from 200 grit sandpaper. A position sensor is attached. Familiar and novel objects were lifted to a height of $6 \mathrm{~cm}$ to be aligned to a vertical marker. 


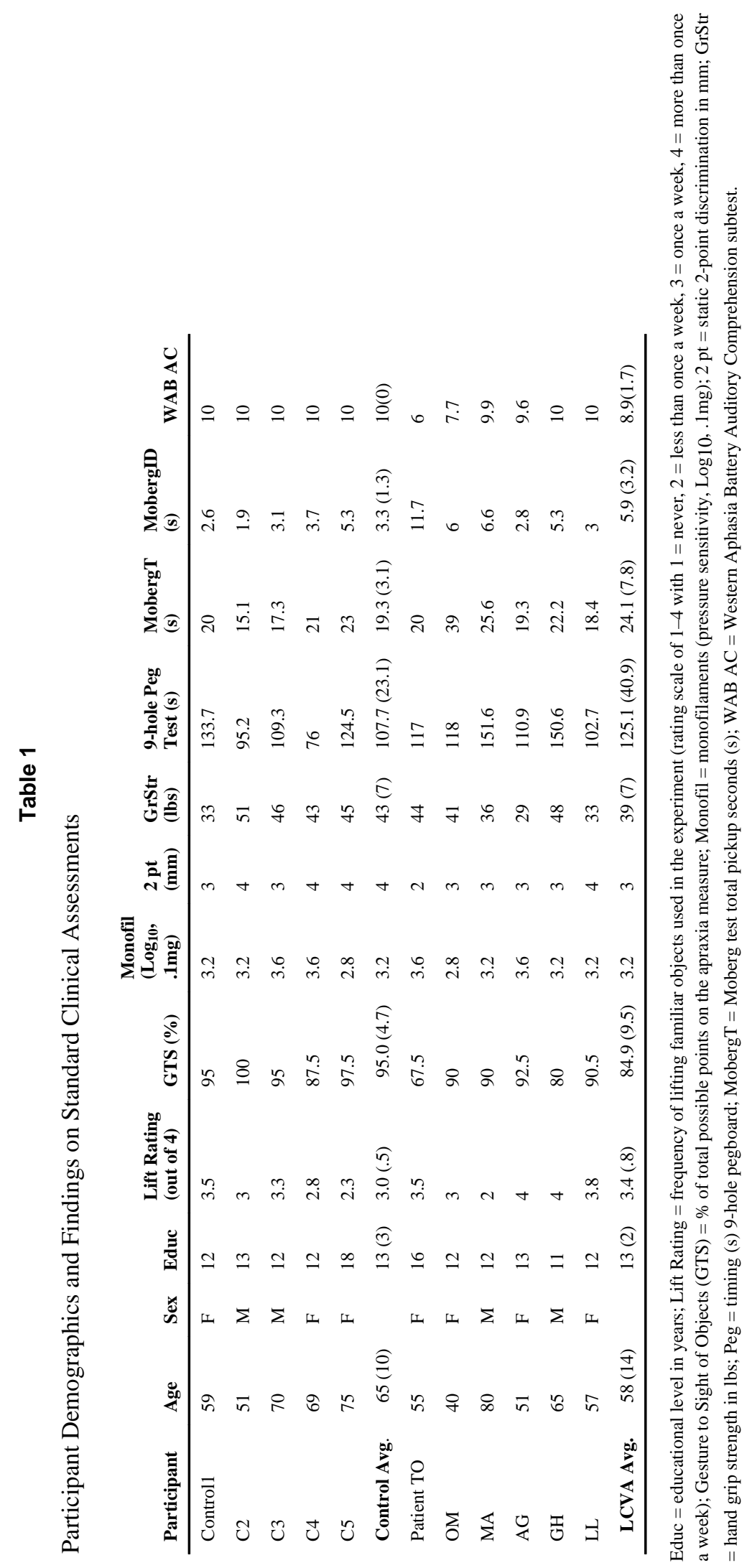

Brain Res. Author manuscript; available in PMC 2011 March 4. 
Table 2

Clinical Characteristics of Participants with LCVA

\begin{tabular}{llll} 
Participant & $\begin{array}{l}\text { Months } \\
\text { Post CVA }\end{array}$ & Brodmann's areas lesioned & $\begin{array}{l}\text { Total voxels } \\
\text { lesioned }\end{array}$ \\
\hline Patient TO & 105 & $21,22,37,39,40,41,42,43,44$ & 39,674 \\
OM & 74 & $1,2,3,7,19,21,22,37,39,40,41,42,43$ & 32,238 \\
MA & 69 & $6,44,47$ & 2,288 \\
AG & 129 & $6,9,45,46$ & 72,991 \\
GH & 75 & $7,22,39,40,41,42$ & 57,196 \\
LL & 101 & $1,2,3,4,9,22,32,34,38,39,40,41,42,43,45,47$ & 27,492 \\
Avg. (sd) & $92(24)$ & & $38,646(24,544)$ \\
\hline * & &
\end{tabular}




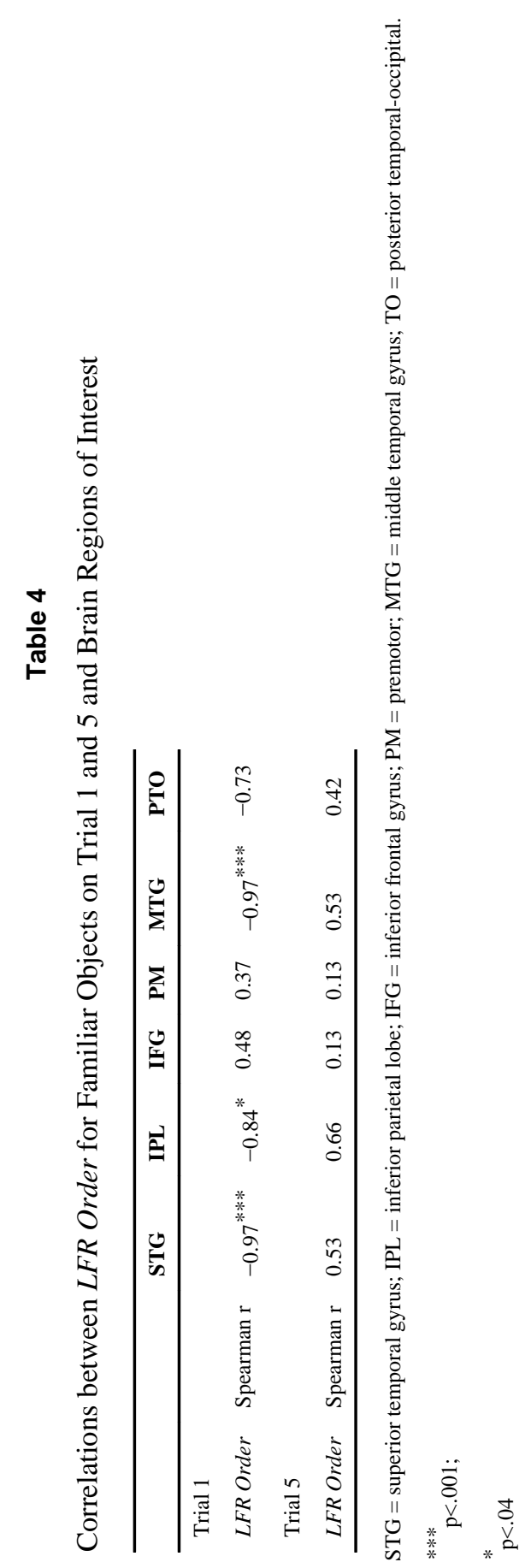

Brain Res. Author manuscript; available in PMC 2011 March 4. 